\title{
Artificial Photosynthesis Beyond Water Splitting for Environmental Remediation
}

Kek Foo Chin, ${ }^{1}$ Miloš Đokić, ${ }^{1}$ and Han Sen Soo ${ }^{1,2, *}$

${ }^{1}$ Division of Chemistry and Biological Chemistry, School of Physical and Mathematical Sciences, Nanyang Technological University, 21 Nanyang Link, Singapore 637371.

${ }^{2}$ Artificial Photosynthesis (Solar Fuels) Laboratory, Nanyang Technological University, 50 Nanyang Avenue, Singapore 639798.

*Correspondence: hansen@ntu.edu.sg (H. S. Soo)

Keywords: artificial photosynthesis, biomass valorization, photoelectrochemical cells, photoredox catalysis, plastics upcycling

\section{Abstract}

Artificial photosynthesis potentially offers solutions to the world's looming energy and environmental crises. Here, we discuss the ongoing challenges of commercializing traditional artificial photosynthetic systems based on water splitting, and highlight the advantages of replacing the water oxidation halfreaction with value-added alternatives, including biomass valorization and plastics upcycling.

\section{Economic Challenges in Using Traditional Artificial Photosynthesis to Overcome the Energy Crisis}

There is growing recognition that the world faces impending energy and environmental crises if we maintain the status quo of relying heavily on fossil fuels. The combustion of fossil fuels produces $\mathrm{CO}_{2}$, a greenhouse gas that traps heat in the atmosphere and is largely responsible for global climate change. Despite this, remarkably little progress has been made to wean the world off fossil fuels. Over a decade after the 2007 Nobel Peace Prize was awarded to the Intergovernmental Panel on Climate Change and Al Gore for bringing attention to the world on anthropogenic climate change, the demand for crude oil is still rising. In fact, the International Energy Agency expects oil demand to continue growing for the next two decades, owing to the population and economic growth of developing countries.

The need for renewable energy storage has spurred research in batteries, supercapacitors, and artificial photosynthesis, with the last being especially attractive since sunlight can be harvested to produce the same chemicals currently derived from fossil fuels. Drawing inspiration from natural photosynthesis, in which water is oxidized to $\mathrm{O}_{2}$ and reduced to energetic hydride equivalents, research in artificial photosynthesis has been preoccupied by the development of photo- and electrocatalytic methods to split water into $\mathrm{H}_{2}$ and $\mathrm{O}_{2}$. However, only $\mathrm{H}_{2}$ is a fuel, whereas $\mathrm{O}_{2}$ is an innocuous by-product that already constitutes around $21 \%$ of the atmosphere, affecting the cost competitiveness of this process. In a recent technoeconomic analysis, the price of $\mathrm{H}_{2}$ produced by using renewable resources to split water is currently around US\$3.50/ $\mathrm{kg}$ and is projected to fall to around US\$2.60/kg by 2030 [1]. To put things in perspective, the price of $\mathrm{H}_{2}$ produced from coal or natural gas is currently around US\$1.30-1.50 [1], and both coal and natural gas reserves are still being uncovered. In other words, if we used artificial photosynthesis to produce $\mathrm{H}_{2}$ fuel solely for the cathodic half-reaction, with no additional value-added reaction for the anodic half-reaction, it would not be economically viable in the foreseeable future without collective action on introducing a sufficiently high carbon tax by governments worldwide. 


\section{Mitigating Climate Change and Overcoming Plastics Pollution Simultaneously?}

As a harbinger of the catastrophes to expect from global climate change, 2019 was a year when uncontrollable wildfires were reported (some concurrently) in the Amazon, Africa, Southeast Asia, North America, and Australia. For instance, over 1 billion native animals were killed in the recent catastrophic fires in Australia. In addition to climate change, another environmental crisis is the proliferation of non-biodegradable plastics in the marine ecosystems. Sir David Attenborough had highlighted in BBC's Blue Planet II series that plastics can now be found in some of the remotest habitats in the world and have accumulated in the notorious Great Pacific and North Atlantic garbage patches, which may remain for centuries.

Some approaches to simultaneously mitigate climate change and these environmental problems are to valorize (add value by chemical transformation of) non-food biomass and to recycle more plastics. In 2015, the full life cycle of fossil fuel-derived plastics was estimated to contribute about $3.8 \%$ of the annual global $\mathrm{CO}_{2}$ emissions, and is projected to rise to around $15 \%$ by 2050 [2]. Notably, $58 \%$ of the plastics were indiscriminately discarded and only $18 \%$ were recycled [2]. However, conscious utilization of renewable energy during the production and active upcycling of plastics can limit the 2050 emissions to 2015 levels or even lower [2].

\section{Artificial Photosynthesis Beyond Water Splitting}

Recently, organic, analytical, and materials chemists have worked together to create high performance, nonaqueous redox flow batteries as potential solutions for grid-scale electrical energy storage [3]. In another example, inorganic chemists, materials engineers, electrical engineers, high energy physicists, and computer scientists assembled to critically assess the historical claims of cold fusion, which offers the promise of virtually unlimited energy [4]. Evidently, interdisciplinary and multi-pronged approaches can provide solutions to some of the world's biggest problems, but the culture in academic research and publishing has created an atmosphere of primacy and exclusivity where competing ideas must be discredited to establish novelty. One example in the field of artificial photosynthesis is the dominance of research on water oxidation due to the ostensible need for a halfreaction that is commensurate in scale for the reductive half-reaction to produce the sizable amounts of fuels needed to supplant fossil fuels. Alternatives to water oxidation have been derided as sacrificial reactions with little scalable potential or limited practicality. This is despite the fact that $\mathrm{H}_{2}$ production alone by artificial photosynthesis is not price competitive with existing production methods from fossil fuels, whereas the oxidation of substrates other than water can provide compensating value to the overall system.

In this context, the Reisner group, the Soo group, and others have tried to synergize concepts from artificial photosynthesis, environmental remediation, and organic syntheses by framing the issue in terms of scalability versus product value (Figure 1). Existing commercialized processes are likely to be in the top left or bottom right quadrants. For instance, petrochemical refining and arguably artificial photosynthetic reactions such as water splitting or $\mathrm{CO}_{2}$ reduction would fall into the top left quadrant, which are processes that have to be conducted in massive scales, but the products are fuels and platform chemicals that must be affordable. Diametrically opposite in the bottom right quadrant lie pharmaceutical compounds, where multistep synthesis, high purity, photoredox catalysis, and biosynthesis are applied to create expensive products on modest scales. In the bottom left quadrant, processes are typically conducted on laboratory scales, such as artificial photosynthesis using sacrificial reagents, which are necessary for knowledge creation and improved fundamental understanding in academia, but typically have low technological readiness levels and show little practical value at the early stages of discoveries. Ideally, we strive to develop artificial photosynthetic 
processes in the top right quadrant, meaning that we should use light to target ubiquitous substrates and still generate valuable products.

Pioneers in the field of artificial photosynthesis including Gray, Nocera, Gratzel, and Meyer have worked on substitutes for water oxidation, the last of whom recently demonstrated a model of seawater oxidation to halogens by creating a system to split $\mathrm{HBr}$ [5]. Seawater is the most abundant source of water and the salts can be transformed into halogens that are platform chemicals used as disinfectants and oxidants. Municipal waste can also be photocatalytically recycled, but the complex mixtures are usually mineralized with little added value [6]. Nevertheless, the valorization of biomass with light, especially non-food agricultural waste, is potentially game changing. A number of groups including Sun's [7], Wang's [8], Stephenson's [9], Reisner's [10], and Soo's [11, 12] have developed homogeneous and heterogeneous catalytic processes to transform lignocellulose-derived substrates into aromatic products or simple fuels like $\mathrm{H}_{2}$. If non-food biomass "waste" such as oil palm residues can be converted into liquid fuels, solvents, or plastic precursors with renewable energy, we can convert slash-and-burn agriculture into a "slash-and-refine" industry to mitigate climate change and environmental pollution.

Besides lignocellulosic biomass, other sources of abundant, recalcitrant, nominal "waste" are discarded non-biodegradable plastics. They are traditionally disposed of in landfills, incinerated, or recycled by melting and reprocessing without changing the chemical composition. To circumvent the energy intensive thermal recycling processes, a breakthrough was reported in 2018 where readily separable and reusable $\mathrm{CdS} / \mathrm{CdO}_{x}$ quantum dot heterogeneous catalysts were employed for the visible light-driven photoreforming of polyethylene terephthalate and other plastics into $\mathrm{H}_{2}$ under ambient temperatures in aqueous conditions [13]. This work was improved after the toxic Cd-based photocatalysts were replaced with a more benign $\mathrm{CN}_{\mathrm{x}} / \mathrm{Ni}_{2} \mathrm{P}$ nanocomposite (Figure 2a) [14]. Nonetheless, both systems required highly alkaline pretreatment to partially hydrolyze the polymers, conversions never exceeded $50 \%$, and the carbons in the plastics were mineralized to $\mathrm{CO}_{2}$ or a complex mixture of carboxylic acids $[13,14]$. The Soo group developed a complementary advance where molecular, base-metal vanadium photocatalysts were employed to unravel polyethylene-based plastics by cascade C-C bond cleavage (Figure 2b) [15]. Full conversion of the plastics was achieved with no pretreatment within the same timeframe, and formic acid was one of the main useful products. However, the photocatalyst is homogeneous and harder to reuse, operates in acetonitrile, and needs modest heating to dissolve the substrates [15].

\section{Sustainable Pathways to Improve the State-of-the-Art in Plastics Upcycling}

By employing an interdisciplinary collaborative approach, the following improvements can map out a path to translate the recent plastics upcycling technologies to more economically viable solutions:

1) The photocatalytic processes should be adapted into photoelectrochemical systems so that electricity from other renewable energy sources can also be used to generate a broader range of products (Figure $2 \mathrm{c}$ ).

2) The activity, selectivity, and operational stability must be improved.

3) There should be minimal pretreatment or heating of substrates.

4) The system should preferably be heterogeneous for operational convenience and can operate in aqueous conditions.

5) A continuous flow process should be engineered to cope with the large production scales.

6) The overall system should remain affordable with no highly toxic constituents. 
Going forward, the implementation of artificial photosynthesis to produce solar fuels at the reduction half-reaction and value-added products for the oxidation half-reaction need not stop at only substrates that are considered as "waste". It would be revolutionary if most oxidation processes in the fine chemicals industries undergo a paradigm shift and are fundamentally redesigned as "acceptorless dehydrogenation" reactions driven completely by renewable energy.

\section{References}

1. Glenk, G. and Reichelstein, S. (2019) Economics of converting renewable power to hydrogen. Nat. Energy 4, 216-222.

2. Zheng, J. and Suh, S. (2019) Strategies to reduce the global carbon footprint of plastics. Nat. Clim. Change 9, 374-378.

3. Hendriks, K.H. et al. (2018) High-performance oligomeric catholytes for effective macromolecular separation in nonaqueous redox flow batteries. ACS Cent. Sci. 4, 189-196.

4. Berlinguette, C.P. et al. (2019) Revisiting the cold case of cold fusion. Nature 570, 45-51.

5. Brady, M.D. et al. (2017) Dye-sensitized hydrobromic acid splitting for hydrogen solar fuel production. J. Am. Chem. Soc. 139, 15612-15615.

6. Chatzitakis, A. et al. (2013) Hydrogen production using a photoelectrocatalytic-enzymatic hybrid system. Catal. Today 209, 60-65.

7. Han, G. et al. (2017) Visible-light-driven valorization of biomass intermediates integrated with $\mathrm{H}_{2}$ production catalyzed by ultrathin Ni/CdS nanosheets. J. Am. Chem. Soc. 139, 15584-15587.

8. Luo, N. et al. (2019) Visible-light-driven coproduction of diesel precursors and hydrogen from lignocellulose-derived methylfurans. Nat. Energy 4, 1-10.

9. Bosque, I. et al. (2017) Redox catalysis facilitates lignin depolymerization. ACS Cent. Sci. 3, 621628.

10. Wakerley, D.W. et al. (2017) Solar-driven reforming of lignocellulose to $\mathrm{H}_{2}$ with a $\mathrm{CdS} / \mathrm{CdO}_{x}$ photocatalyst. Nat. Energy 2, 17021.

11. Gazi, S. et al. (2017) Kinetics and DFT studies of photoredox carbon-carbon bond cleavage reactions by molecular vanadium catalysts under ambient conditions. ACS Catal. 7, 4682-4691.

12. Gazi, S. et al. (2015) Selective photocatalytic $C-C$ bond cleavage under ambient conditions with earth abundant vanadium complexes. Chem. Sci. 6, 7130-7142.

13. Uekert, T. et al. (2018) Plastic waste as a feedstock for solar-driven $\mathrm{H}_{2}$ generation. Energy Environ. Sci. 11, 2853-2857.

14. Uekert, T. et al. (2019) Photoreforming of nonrecyclable plastic waste over a carbon nitride/nickel phosphide catalyst. J. Am. Chem. Soc. 141, 15201-15210.

15. Gazi, S. et al. (2019) Visible light-driven cascade carbon-carbon bond scission for organic transformations and plastics recycling. Adv. Sci. 6, 1902020. 


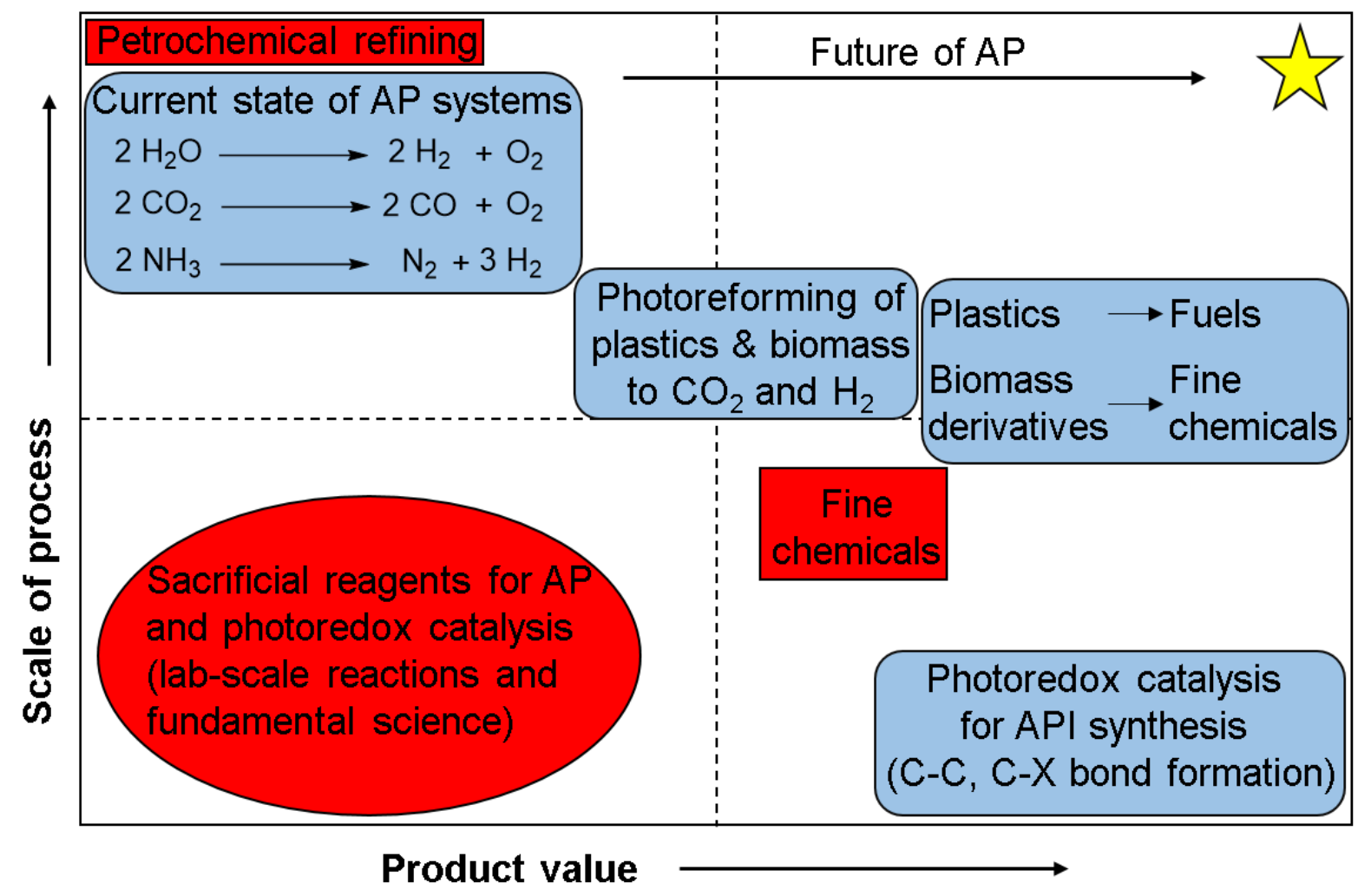

Figure 1. Assessment of chemical processes by comparing their scalabilities versus the product values. Our goal is to develop scalable and economically attractive artificial photosynthetic (AP) processes in the upper right quadrant (marked by the yellow star). The blue boxes represent more eco-friendly processes that can already be achieved by the scientific community, whereas the red boxes indicate the less sustainable status quo. Adapted from [15] with permission. 
(a)

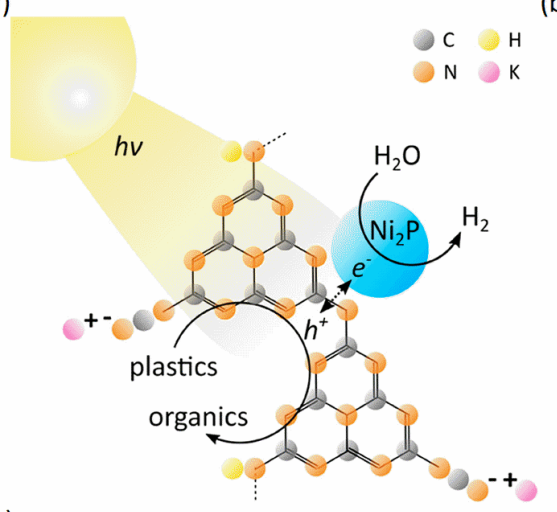

(b)

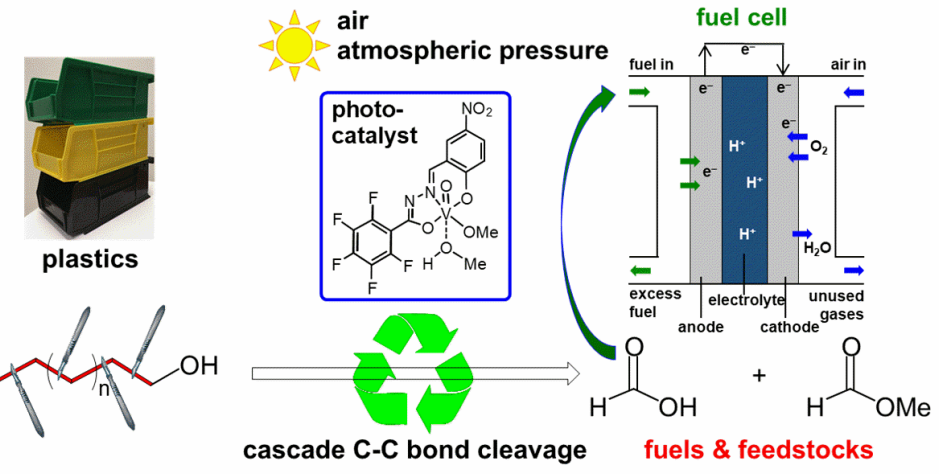

(c)

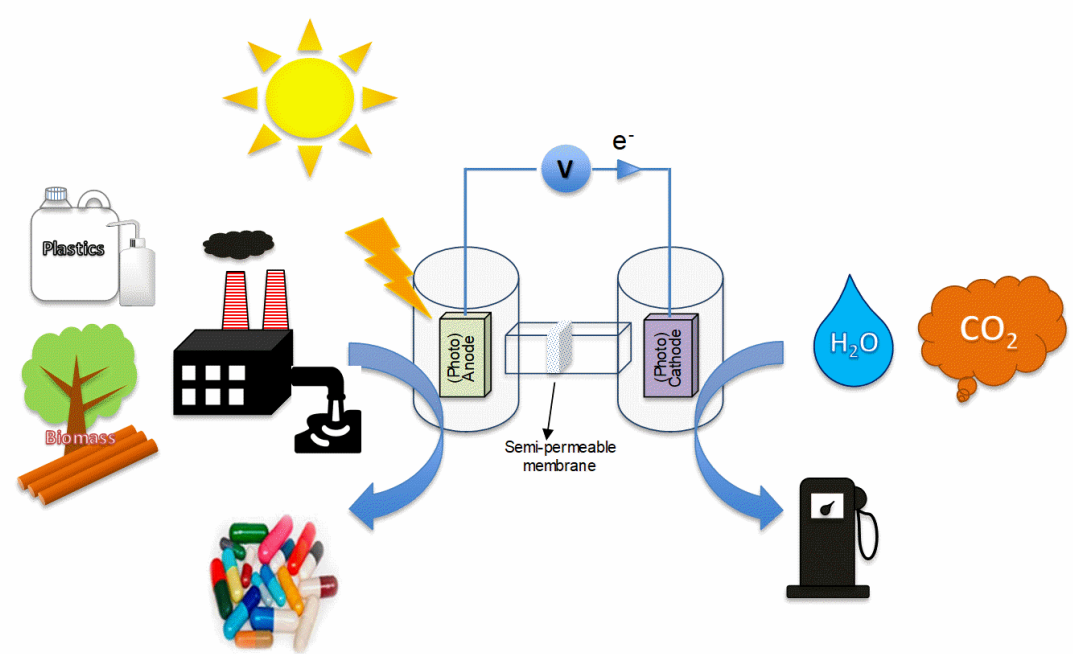

Figure 2. Existing approaches and future advances for repurposing plastics, biomass, and pollutants into solar fuels and valuable chemical feedstocks. (a) Simplified reaction scheme of the polymer photoreforming process using a carbon nitride/nickel phosphide photocatalyst. Reproduced with permission from [14]. (b) Repurposing non-biodegradable polyethylene into formic acid and formates via cascade carbon-carbon bond cleavage driven by visible light and a vanadium photocatalyst. Reproduced with permission from [15]. (c) A more general and versatile photoelectrochemical cell for pollutant degradation, biomass valorization, and plastics upcycling, driven by light and electricity from renewable energy sources. 